\title{
Multilinguales
}

1 | 2013

Pratiques littéraires, linguistiques, pédagogiques,

didactiques et médiations culturelles contemporaines

\section{Autour de la parémiologie contrastive : Le concept d'« énoncé culturel »}

Around "paremiologia": the concept of cultural statement

Mame Couna Mbaye

\section{(2) OpenEdition}

Journals

Édition électronique

URL : https://journals.openedition.org/multilinguales/3396

DOI : 10.4000/multilinguales.3396

ISSN : 2335-1853

Éditeur

Université Abderrahmane Mira - Bejaia

Édition imprimée

Date de publication : 1 juin 2013

Pagination : 169-179

ISSN : 2335-1535

Référence électronique

Mame Couna Mbaye, «Autour de la parémiologie contrastive : Le concept d'« énoncé culturel » », Multilinguales [En ligne], 1 | 2013, mis en ligne le 01 juin 2013, consulté le 30 juin 2021. URL : http:// journals.openedition.org/multilinguales/3396; DOI : https://doi.org/10.4000/multilinguales.3396

Ce document a été généré automatiquement le 30 juin 2021.

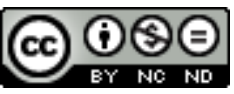

Multilinguales est mise à disposition selon les termes de la Licence Creative Commons Attribution -

Pas d'Utilisation Commerciale - Pas de Modification 4.0 International 


\title{
Autour de la parémiologie contrastive : Le concept d'« énoncé culturel»
}

\author{
Around "paremiologia": the concept of cultural statement
}

\author{
Mame Couna Mbaye
}

1 L'article que nous présentons ici est le fruit d'une réflexion que nous avons entreprise en travaillant sur les proverbes en français, en espagnol et en wolof. En effet, nous appartenons à ces différents univers : à l'espagnol de par notre formation universitaire, au français parce que c'est la langue officielle de notre pays d'origine (Sénégal) et au wolof parce que c'est notre langue maternelle. Nous avons ainsi éprouvé la nécessité de travailler sur des proverbes appartenant aux trois langues, car nous avons constaté que malgré la différence des langues, nous y retrouvons certaines similitudes de sens et de fonctionnements linguistiques même si les référents ne sont pas toujours les mêmes. Le but de cette entreprise est une tentative de mise en évidence d'universaux culturels malgré l'éloignement des langues, en l'occurrence les langues espagnole et française (deux langues indo-européennes) face à la langue wolof (une langue africaine de la famille nigéro-congolaise).

2 Cependant, lors de la constitution de notre corpus de proverbes dans ces trois langues, auprès de différentes sources, nous nous sommes aperçue qu'il existait plusieurs dénominations pour désigner ce que nous considérions comme proverbe, que ce soit dans une même langue ou d'une langue à une autre. Nous nous sommes alors posée la question de savoir quelle notion pourrait englober l'ensemble des énoncés constitutifs de notre corpus dans les trois langues considérées, sachant que le proverbe peut être défini comme l'ensemble des énoncés sentencieux appartenant à la sagesse populaire d'un peuple donné. Ces premières observations justifient notre choix de parler d'énoncés culturels (E.C.) ${ }^{1}$ pour désigner l'ensemble des énoncés sentencieux dans une même langue. 


\section{Au commencement était le proverbe}

3 Signalons tout d'abord que le but de ce travail n'est pas de démontrer ce qu'est un proverbe, mais de préciser ce que nous entendons par le concept E.C.. Nous essayons ainsi d'expliciter notre choix concernant la dénomination E.C. dans cet article car, dans les différentes sources utilisées pour la constitution d'un corpus de proverbes dans les langues française, espagnole et wolof, ce type d'énoncé est désigné par différents termes.

Pour ce qui est des E.C.. de la langue française, nous avons utilisé le Dictionnaire des proverbes, sentences et maximes (Maloux, 1960) et Le livre des proverbes français ... (Le Roux de Lincy, 1859), où le proverbe est assimilé aux notions de maxime et de sentence. Dans le cas de la langue wolof, les sources utilisées sont Sagesse africaine: proverbes wolof (Samb et Ndao, 1983) et Léébu, proverbes wolof (Cribier et al., 1986). Nous remarquons ici que c'est le vocable « léébu » qui est traduit en français par « proverbe ».

5 En ce qui concerne la langue espagnole, dans les trois sources utilisées, à savoir Refranero español, Antología de refranes populares y cultos de la lengua castellana explicados y razonados (Fernández, 1987), Refranero temático español (Doval, 1997) et Refranes, proverbios y sentencias (Calles Vales, 2007), l'omniprésence de la notion de «refrán » à travers son pluriel « refranes » et le terme «Refranero » qui traduit une collection de refranes, exige quelques éclaircissements. C'est pourquoi, avec José Calles Vales, nous nous intéressons aux origines de cette notion de « refrán » en Espagne :

En las antiguas canciones medievales los trovadores solían incluir uno o varios versos que se repetían al final de cada estrofa. Es lo que, en la actualidad, conocemos como "estribillo". Aquellas primeras muestras poéticas utilizaban como estribillos breves sentencias populares, rimadas o en forma de proverbios. Los trovadores provenzales llamaron a esta secuencia poética "refrain", y éste es el origen de la palabra española "refrán". ${ }^{2}$

Le terme espagnol "refrán» était utilisé pour des sentences populaires et/ou des proverbes de cette langue. Selon le parémiologue ${ }^{3}$ français Michel Quitout (2002), la parémiologie occidentale regroupe le proverbe, la sentence, la locution proverbiale, le dicton, la maxime, le slogan, l'adage, le précepte, l'aphorisme, l'apophtegme, la devise et le wellérisme. Chacune de ces douze notions est appelée parémie.

7 Cependant, même si la parémie est l'hyperonyme de tous ces énoncés sentencieux, chaque chercheur peut l'orienter selon les buts qu'il fixe à sa recherche. Les caractéristiques à considérer pour cerner cette notion de parémie découlent ainsi des caractéristiques communes aux notions retenues par le chercheur parmi celles qu'elle englobe. Ainsi, la notion de parémie comprend l'ensemble des énoncés sentencieux répertoriés dans une ou plusieurs langues données (proverbes, sentences, adages, etc.), mais par consensus méthodologique, comme le signale Francis Rodegem (1984) :

J'aimerais vous faire une proposition, c'est de nous [spécialistes en parémiologie] mettre d'accord sur une terminologie commune. Quel vocable convient le mieux pour désigner l'ensemble des formules consacrées par l'usage, qu'on appelle généralement "proverbes"? Sans préjuger des résolutions finales de notre colloque, mais pour éviter les ambiguïtés, je réserverai le terme de parémie aux énoncés sentencieux pris globalement. Nos échanges et nos mises en commun permettront, je l'espère, de définir les notions avec plus de rigueur. (121)

8 L'hésitation manifeste des spécialistes à cerner les contours de la notion de parémie, à l'instar de Francis Rodegem, nous pousse à adopter une autre notion qui nous permet 
d'englober l'ensemble des énoncés sentencieux de plusieurs langues sans courir le risque de convoquer un panorama de définitions pour chacune des notions que renferme la parémie.

Il convient aussi de signaler qu'étymologiquement, le terme parémie vient du grec «paroemia » et que ce mot signifie proverbe. Ce qui fait qu'il nous semble tout aussi légitime pour un chercheur de ne considérer que les proverbes, quand il parle de la discipline (parémiologie) ou du terme (parémie). En effet, le terme "paroemia (proverbe) désignait en grec un énoncé anonyme considéré comme un héritage collectif et différent en cela de la "gnômé" (maxime), qui était, elle, la réflexion et la composition d'un homme sage comme Aristote ». (Schapira, 1999 : 57)

Outre cette mise au point sur la difficulté liée de la délimitation rigoureuse de ces notions de proverbes, de parémie, de "refrán ", etc., il faut également signaler que toute tentative de délimitation de ces notions serait un travail à la fois fastidieux et inutile (Combet, 1991 : 15), comme en témoignent leurs nombreuses définitions par les lexicographes et les étymologistes. Louis Combet (1991: 15) invite à suivre la conception de certains auteurs espagnols qui ne distinguent pas le terme « proverbio " de « refrán». Les titres de ces deux recueils (respectivement ancien et moderne), consacrés à ces énoncés, le prouvent: Refranes o proverbios (Nuñez, 1555), Refranes, proverbios y sentencias (Calles Vales, 2007).

11 En effet, si dans le premier ouvrage, Refranes o proverbios, l'assimilation de « refranes » à "proverbios» est perceptible dès le titre, en ce qui concerne le second ouvrage, Refranes, proverbios y sentencias, la notion de " refrán » est saisie comme un hyperonyme des notions de "proverbio » et de "sentencia ». Ceci est saisissable dès la première page de couverture : «REFRANES » est mis en évidence à travers une écriture en gras et en majuscule sur toute la largeur de la page, alors que les vocables «PROVERBIOS y SENTENCIAS» sont en majuscule, mais plus petites que les premières et sans gras, comme pour signifier que le terme « refranes » renfermant ceux de « proverbios » et de « sentencias » est plus important. Ce qui justifie selon son auteur la difficulté qui réside dans une tentative de proposer des différenciations entre ces différentes notions.

C'est la raison pour laquelle, dans cet ouvrage, le principal critère de recueil de ces énoncés est leur regroupement par thème.

Pour Francisco Rodríguez Marín (1926), le refrán serait «un dicho [...] popular, sentencioso [...] y breve [...] de verdad (proverbium), generalmente simbólico (parábola) y expuesto en forma poética [...], que contiene una regla de conducta (adagio) u otra cualquiera enseñanza $[. ..] »^{4}(x v i i i-x i x)$.

14 En effet, la présence de ces différentes notions qui composent la définition de "refrán », à savoir qu'il est à la fois un "dicho popular », "sentencioso », doté d'une vérité qui lui donne son statut de "proverbium» (proverbe), énonçant une règle de bonne conduite qui justifie son caractère de "adagio », etc., montre bien la difficulté liée à la délimitation des contours du "refrán» par rapport à toutes ces notions contiguës.

Dès lors, il convient de préciser que ce qui nous importe le plus dans ce type d'énoncés, c'est qu'ils soient des créations culturelles caractérisées essentiellement par certains critères comme ceux de leur autorité, leur supposée-vérité et leur permanence naturelle. 
16 Eu égard, à ces considérations préliminaires, il est à noter qu'il nous faut mettre en relief un consensus entre les langues espagnole, française et wolof du point de vue à la fois dénominatif et référentiel.

Pour ce qui est de la langue wolof, nous avons vu qu'il existait le terme « léébu » qui est rendu en français par "proverbe». Il est important de préciser que ce vocable, "proverbe ", se réfère à une acception purement française et qu'elle ne rend pas compte de la totalité de ce qu'en wolof, on considère comme proverbe. Ainsi, la traduction de « léébu » par " proverbe » n'est pas exacte, comme le signale Cheik Aliou Ndao (1983) :

J'ai traduit le mot « Léébu » par proverbe, mais en fait, il est beaucoup plus complet.

[...] Il est difficile de faire passer la saveur du terroir dans une langue étrangère. J'ai seulement pensé que ces dictons, sentences, proverbes, etc., devaient être mis à la portée d'un plus grand nombre de publics. (3)

Avec les affirmations de Cheik Aliou Ndao, nous remarquons que nous ne pouvons pas assimiler la notion de proverbe à celle de "léébu ", telle qu'elle est perçue en wolof. Ici, le concept d'énoncé culturel ${ }^{5}$ nous semble plus à même de rendre compte de la notion de « léébu » telle que cette dernière est envisagée dans cet ouvrage (dictons, sentences, proverbes, etc.).

En effet, cette notion de "Léébu " revient à dénommer ce que la parémiologie occidentale désigne par le terme de parémie, tout en précisant que, dans le cas de la société wolof, il n'existe pas de contestation de cette notion de «léébu » telle qu'on peut le remarquer en Occident avec le wellérisme. Pour plus de précision, il convient aussi de nous arrêter sur le vocable wolof «léébkat» formé à partir du mot wolof « lééb » qui signifie conte et dont est tiré le vocable « léébu ». Dans son acception, le terme wolof «Léébkat » est un des trois termes qui regroupent le seul mot français conteur. En effet, au Sénégal, les conteurs se répartissent en trois groupes à savoir :

- les «Léébkat ", terme qui signifie donc conteur en wolof. Le terme « lééb " traduit conte en français et le suffixe « kat » sert à former les noms d'agent ou d'artisan d'un métier. Ce sont des conteurs professionnels formés à l'art de la parole. Ils sont à distinguer des Griots, car ils sont issus de toutes les couches de la population sénégalaise. En général, ils acquièrent leur formation dans des compagnies de théâtre traditionnel où le récit, la musique et la danse sont liés. Mais, certains « Léébkat » sont formés au sein de leurs propres familles auprès des grands-parents et des parents.

- Les Griots : ils sont appelés «Gewël » en wolof, «Gawlo» en peul, et «Djeli » en mandingue. C'est un groupe social anciennement caractérisé par l'hérédité et l'endogamie. Ils sont désignés comme des artisans de la parole. Ils sont artistes, historiens de l'oralité et généalogistes.

- Les «Borom xam-xam»: eux sont les maitres du savoir. Ils transmettent leur enseignement à travers les mythes, les contes et la réflexion philosophique. L'un des plus connus est Kocc Barma Fall, philosophe et conteur wolof. ${ }^{6}$

Dans cette classification, nous remarquons que même si le mot wolof "léébkat " provient du mot « lééb ", il ne rend pas l'idée de proverbe comme dans sa traduction en français. De la même manière, nous découvrons aussi que le groupe de mots wolof «borom xam-xam », qui signifie philosophe, est octroyé à Kocc Barma Fall à qui l'on attribue une grande majorité des proverbes wolof. ${ }^{7}$ Toutefois, ces énoncés ne sont pas que des proverbes dans le sens restrictif que lui donne le français à savoir qu'il est à distinguer de la sentence, de la maxime, de l'adage, etc., tels qu'ils sont répertoriés 
dans la parémiologie occidentale. Ce qui est intéressant de retenir ici, nous semble-t-il, c'est qu'au Sénégal, ces contes ne sont pas racontés uniquement pour distraire; il s'agit, dans le cas des «Borom xam-xam » et des «Léébkat », d'un moyen pédagogique pour transmettre des règles de morale sociale, à travers des messages codés soumis à l'assistance qui se doit de les déchiffrer. C'est dans ce même registre que les «Gewël » participent à la fois à la stimulation et à l'inculcation des valeurs traditionnelles aux enfants et aux jeunes adultes, mais aussi au rappel des règles de la vie en société à tous les membres de la communauté, en chantant le courage des ancêtres, les vertus de la dignité et de la loyauté.

21 En wolof, cette notion de « léébu » désigne donc l'ensemble des énoncés attribués à des «Borom xam-xam » (philosophes), des « Gewël » (griots), des « Léébkat » identifiés ou non. Ces énoncés doivent renfermer certaines caractéristiques, à savoir qu'ils soient de nature permanente et véridique parce que renfermant des préoccupations collectives dont les buts principaux sont entre autres, la consolidation des relations entre les membres de la communauté, et avec le monde extérieur.

\section{Vers une définition du concept « énoncé culturel » (E.C.)}

Cette différenciation dans la signification notionnelle entre les différentes langues, à savoir le wolof, l'espagnol et le français, nous pousse donc à adopter à la place de toute autre notion approximative (proverbe), ou générique (la parémie dans le cadre de l'Occident), celle de la d'E.C. qui, de notre point de vue, est plus opératoire. Elle permet, en effet, d'englober l'ensemble des énoncés sentencieux de plusieurs langues. C'est le cas des exemples suivants :

Espagnol : De tal palo, tal astilla (« Tel bois, telle écharde »).

Français : Tel père, tel fils

Wolof : Këll bu ne am na mberoom (« Chaque calebasse a son couvercle »).

Ces trois proverbes ont le même sens et signifient dans chacune de ces langues.

Pour ce qui fonde le concept d'E.C. dans notre recherche, précisons que cette désignation se justifie essentiellement par les caractéristiques que partagent les parémies et d'autres énoncés sentencieux (créations culturelles), à savoir qu'elles renferment un principe de vérité, d'autorité et de permanence naturelle. Ces trois caractéristiques se justifient, quant à elles, par le fait que les E.C. expriment les préoccupations (sur tous les plans) des membres de la communauté.

Il faut aussi signaler que dans le cas de l'Occident, la différenciation de ces désignations (proverbes, sentences, dictons, adages, etc.) se fonde généralement au niveau d'une "délimitation de typologies à partir de critères fondamentalement linguistiques (repérage de structures lexicales, morphosyntaxiques, et de relations sémantiques)» (Queirós Fréjaville, 2003 : 27). Nous ne comptons pas, quant à nous, définir le concept E.C. à partir de caractéristiques linguistiques (description linguistique des différents éléments qui composent les E.C.). Une analyse ne se limite pas uniquement à un point de vue purement linguistique, il s'agit aussi de prendre en compte la dimension culturelle, c'est-à-dire, le statut des énoncés au sein de la communauté dans laquelle ils sont nés. 
25 Avec Gérard Leclerc (1996: 8), nous admettons que les E.C. sont fondés sur le principe d'une " autorité énonciative », c'est-à-dire qu'ils induisent une persuasion et des effets de croyance, comme c'est le cas dans les exemples suivants :

Espagnol : Tanto va el cántaro a la fuente que al fin se rompe (Tant va la cruche à l'eau qu'à la fin elle se casse ).

Français : Tant va la cruche à l'eau qu'à la fin elle se casse.

Wolof : Lu ëpp tuuru (Tout ce qui est trop déborde).

26 Á la première caractéristique, qui est celle de leur autorité, s'ajoute celle du principe de vérité qu'ils prétendent énoncer, et qui semble aussi favoriser ce statut à la fois d'autorité et d'atemporalité dont jouissent ces énoncés particuliers que nous avons dénommés, pour ces raisons, E.C..

Ces trois caractéristiques (autorité, supposée-vérité, permanence naturelle) se combinent selon un modèle d'interdépendance successive qui se justifie par le fait que les E.C. renferment des préoccupations collectives :

Espagnol : Quien ríe el último reirá mejor (Qui rit le dernier rira mieux).

Français : Rira bien qui rira le dernier.

Wolof: Ku nekk ci xare do ree kunu jam (Celui qui est dans une bataille ne doit pas se moquer de celui qui vient d'être tué).

28 Après cette mise au point visant à justifier notre choix d'utiliser le concept d'E.C., il nous semble important et même nécessaire d'insister sur quelques points.

L'autorité d'un E.C. ne se loge pas uniquement au sein de ses propos internes (obtenus en combinant les référents qui composent l'énoncé), ni externes (obtenus en considérant la signification métaphorique de l'énoncé), même si ces deux caractéristiques sont fondamentales dans l'acceptation de la vérité d'un E.C.. Cette autorité va au-delà de ces considérations car les E.C. sont, de notre point de vue, protégés par l'ensemble de leur communauté de naissance.

30 Á présent, revenons d'une manière beaucoup plus ample sur les trois critères que nous avons retenus (autorité, prétendue-vérité et permanence naturelle) pour cerner notre concept d'E.C..

31 Pour la définition de l'autorité, nous allons faire appel à Gérard Leclerc (1996) qui distingue deux types d'autorité séparés par leur temporalité, leur désignation et leur nature :

L'autorité n'est plus ce qu'elle était. Autrefois principe majeur de légitimation des discours, elle signifie aujourd'hui le mode d'existence des pouvoirs légitimes. Elle fut pendant des siècles une propriété des énoncés, une qualité de certaines idées, un privilège de certains penseurs. Elle est aujourd'hui un élément des institutions, le principe symbolique des pouvoirs. L'autorité fut pendant deux millénaires un concept philosophique; elle désigne depuis le siècle dernier un concept sociologique. (7)

D'après les définitions de ces deux types d'autorité, il convient de faire la remarque que les E.C., dans notre acception, appartiennent à la forme de l'autorité énonciative du fait de leur atemporalité et de la nature de leur statut qui leur confère croyance et crédibilité, pour reprendre les termes de Gérard Leclerc. Selon lui, l'autorité énonciative est composée de l'autorité des penseurs et de l'autorité culturelle des textes (8). 
Dans le cadre de ce présent article, c'est cette autorité énonciative qui nous intéresse, dans la mesure où elle regroupe l'autorité des penseurs (qui créent ces énoncés) et l'autorité culturelle des textes (ces énoncés restent indiscutables, du moins, au sein de leur communauté de naissance).

Cependant, selon Gérard Leclerc, cette autorité a subi une dégradation dans l'histoire en cédant la place au principe de la rationalité (9). En effet, ce principe de rationalité dans le cadre des E.C. peut s'expliquer, pour ce qui est de l'Occident, par une contestation parodique plus connue sous le nom de wellérisme. Et pourtant, selon Francis Rodegem (1986 : 126), le wellérisme avait fait son apparition depuis le III siècle avant notre ère.. Dans cette perspective, l'autorité inviolable des E.C. a été mise en cause depuis fort longtemps. Il faut donc chercher une autre caractéristique qui puisse justifier leur survie.

5 Selon Gérard Leclerc (1996), «l'autorité d'un énoncé ou d'un auteur, c'est sa crédibilité, la probabilité qu'il soit véridique. Cette crédibilité est évaluée par le lecteur, en principe, d'abord et avant tout sur la base des mérites propres de l'énoncé et de l'auteur » (13).

En ce qui concerne la question de la vérité relative à ces E.C., elle se loge dans le contenu du message que véhicule un énoncé comme l'indique Gérard Leclerc: "L'autorité entretient quant à elle un lien intime avec la question de la qualité et de la vérité de l'énoncé» (11). La question que nous nous posons est celle de savoir où se situe la vérité d'un énoncé ? Gérard Leclerc y répond :

Avec la disparition des garants transcendants de la vie humaine et de sa signification - car avec Dieu disparaitt la garantie transcendante de la Vérité - il n'est plus d'autres garanties que celles qui sont logées dans l'immanence de la vie psychologique et sociale. La garantie - relative - de la vérité réside dans la crédibilité différentielle des énonciateurs sociaux et culturels (des spécialistes, des intellectuels, des hommes politiques), dans l'autorité purement immanente de l'esprit individuel, et dans ses bases institutionnelles, symboliques et psychologiques. (12)

Dans ce cas, les conditions d'une vérité sont logées dans le cercle de l'expérience humaine, c'est-à-dire que les conditions d'acceptation d'un énoncé doivent relever de considérations empiriques, car la notion de vérité peut être mise en cause dès l'instant que se pose la question de la croyance. Dès lors, il n'est plus question de vérité tout court, mais de vérité tangible. Or, tous les E.C. ne reflètent pas des vérités tangibles.

Considérons les E.C. espagnol et français suivants, dans leurs significations purement littérales :

"Quien va a Sevilla pierde su silla » et "Qui va à la chasse perd sa place », que nous avons commentés plus haut. Ces affirmations ne requièrent aucune vérité tangible. Mais si la vérité admet comme définition l'accord de la pensée avec la chose, selon Jules Lachelier ${ }^{8}$, le message d'un énoncé doit simplement admettre l'accord de la pensée avec ce qui est énoncé. C'est dans cette perspective que les E.C. «Quien va a Sevilla pierde su silla » (Qui va à la chasse perd sa place) et «Qui va à la chasse perd sa place " peuvent avoir les statuts qu'on leur connaît au sein de leurs langues respectives. C'est pour cela que Gérard Leclerc affirme :

La vérité d'un énoncé se marque rarement d'elle-même dans l'évidence de ses raisons claires et distinctes, comme le sont les vérités mathématiques. L'évidence logique est rarement le critère unique et premier de la crédibilité d'un énoncé nouveau. [...]. Elle est l'enjeu d'une évaluation probabiliste, qui fait certes référence 
à sa crédibilité interne (à l'évidence des raisons), mais aussi à sa crédibilité externe (la fiabilité de son énonciateur). (13)

\section{et le statut de celui qui le dit. Ce qui est dit doit pouvoir être vérifié par la rationalité ou par l'expérience commune. Le statut de celui qui l'énonce doit être reconnu. Ce deuxième point concernant la reconnaissance attribuée à l'auteur d'un énoncé est plus que déterminant dans la considération des E.C. : \\ L'évaluation de la vérité d'un énoncé suppose donc souvent celle de sa source, des caractéristiques psychologiques de l'auteur supposé, des caractéristiques sociologiques de l'institution qui le patronne. [...] La recherche de la vérité suppose le dialogue, la libre discussion, l'examen des raisons données à son discours par l'interlocuteur; elle est libre confrontation d'énoncés et d'énonciateurs. (Leclerc, $1996: 13$ - 49)}

que la notion de vérité, dans ce cas, fait appel à deux crédibilités : ce qui est dit

41 Á partir de ces deux remarques, et surtout de la dernière, quant à la vérité des E.C., nous percevons la raison ou les raisons pour laquelle/lesquelles, les E.C. se rangent au départ dans la catégorie des énoncés véridiques. Si la recherche de la vérité suppose le dialogue, la libre discussion et l'examen des raisons, dans la mesure où les propos d'un E.C. reflètent la pensée d'une collectivité, le problème de la discussion ou du dialogue est dépassé. Alors, la vérité d'un E.C. est préétablie. Un E.C. pris dans sa singularité renferme toujours une vérité. Ainsi, nous pouvons affirmer qu'entre les deux crédibilités qui régissent le statut de vérité d'un E.C., à savoir sa crédibilité interne qu'évoque l'évidence de ses propos et sa crédibilité externe qu'engendre la fiabilité de son énonciateur, la seconde crédibilité l'emporte sur la première par le poids qu'elle acquiert de l'ensemble d'une communauté donnée. Il convient donc de noter la primauté de la crédibilité externe d'un E.C. sur sa crédibilité interne, laquelle s'explique par le fait que la vérité d'un E.C. est incontestable au sein de la communauté qui l'a créé. Cette seule considération donne le statut de vérité à un E.C. (du moins au sein de sa communauté linguistique et culturelle de naissance).

Cependant, selon Gérard Leclerc, "la vérité n'est pas l'orthodoxie (la vérité officielle) ou la tradition (la vérité reçue), elle est l'assentiment intérieur de l'individu à sa propre pensée » (157). C'est peut-être pour cela que l'on peut contredire les propos d'un E.C. dans le discours ou hors discours. Mais, contredire les propos d'un E.C. donne-t-il autant de poids aux nouveaux propos et à celui qui les émet ou diminue-t-il le poids des propos de l'énoncé contredit?

Pour répondre à cette question, proposons l'exemple de la parole du vieillard Dogon que nous rapporte Gérard Leclerc »Bien qu'elle émane d'un individu remarquable [le vieillard Dogon], sa parole possède plus de valeur d'être celle de la tradition anonyme que l'œuvre d'un simple individu»(374). Nous remarquons, à la lecture de cette citation, qu'au-delà de la vérité qui peut émaner d'un E.C., sa seule provenance de la tradition anonyme la rend fiable. C'est ce qui explique que des E.C. puissent susciter des contreexemples. Mais la censure d'un E.C. équivaut à une condamnation de la pensée de tout un peuple. Ainsi, dans une communauté donnée, celui qui contredit ou émet des critiques à l'égard d'un E.C., contredit et critique l'ensemble de la communauté à laquelle il appartient.

Un autre aspect du statut inviolable de l'E.C., malgré les remous de la modernité, est celui de sa permanence naturelle évoquée d'une manière indirecte par Gérard Leclerc :

Dans la Modernité tout individu est censé avoir une opinion personnelle, pouvoir légitimement énoncer par lui-même. Dans les cultures de la tradition, l'individu 
quelconque n'énonce pas par lui-même, mais s'en remet à l'autorité des Anciens.

(343) Dans cet article, et ceci par rapport aux langues considérées, les énoncés sentencieux sont plus connus sous les noms de "proverbio ", "refrán ", "sentencia » en langue espagnole, « léébu » en langue wolof et " proverbes », « sentence » en langue française. Pour formuler des bases théoriques au concept d'E.C., nous avons mis en avant les critères d'autorité, de vérité, de permanence naturelle ou d'atemporalité.

\section{BIBLIOGRAPHIE}

CALLES VALES J., Refranes, proverbios y sentencias, Madrid, Editorial Libsa, 2007.

COMBET L., Recherches sur le « Refranero » castillan, Paris, Les Belles Lettres, 1991.

Doval G., Refranero temático español, Madrid, Ediciones del Prado, 1997.

CRIBIER J. et al., Léébu, proverbes wolof, Paris, Edicef, 1986. 
FERNÁNDEZ M., Refranero español, Antología de refranes populares y cultos de la lengua castellana explicados y razonados, Madrid, Burdeos, 1987.

LECLERC G., Histoire de l'autorité : L'assignation des énoncés culturels et la généalogie de la croyance, Paris, Presses Universitaires de France, 1996.

LE ROUX DE LINCY, A., Le livre des proverbes français: Précédé de recherches historiques sur les proverbes français et leur emploi dans la littérature du Moyen Âge et de la Renaissance, Paris, 1859, Tome 1, p. VIILXXXIV.

MALOUX M., Dictionnaire des proverbes, sentences et maximes, Paris, Larousse, 1960.

NÚÑEZ H., Refranes o proverbios en romance que nuevamente coligió y glosó el comendador Hernán Núñez, Salamanca, 1555.

QUEIRÓS FRÉJAVILLE, Rosa María : « Le concept d'expression figée dans le langage spécialisé », Stéréotypes linguistiques, Textures : Cahiers du CEMIA, $\mathrm{n}^{\circ}$ 10, Université Lumière Lyon 2, 2003, p. 27-46.

QUITOUT M., Proverbes et énoncés sentencieux, Éditions L’Harmattan, 2002.

RODEGEM F., « La parole proverbiale », In Suard Françoise (dir.), Richesse du proverbe, Université de Lille III, 1984, p. 121-135.

RODRÍGUEZ-MARÍN F., Más de 21.000 refranes castellanos no contenidos en la copiosa colección del maestro Gonzalo Correas. Allególos de la tradición oral y de sus lecturas durante más de medio siglo (1871-1926, Madrid, Imprenta de la Revista de Archivos, Bibliotecas y museos, 1926, p. xviii-xix.

SAMB E.A.N., NDAO C.A., Sagesse africaine : proverbes wolof, Dakar, Imprimerie Saint-Paul, 1983.

SCHAPIRA C., Les stéréotypes en français : proverbes et autres formules, Paris, Ophrys, 1999.

\section{NOTES}

1. Notons que pour tout le reste du texte, nous avons convenu d'adopter désormais l'abréviation E.C. pour désigner ce concept.

2. Traduction : Dans les vieilles chansons médiévales, les troubadours avaient l'habitude d'insérer un ou plusieurs vers répétés après chaque strophe. C'est ce que nous connaissons aujourd'hui sous le nom de «refrain ». Ces premiers échantillons poétiques utilisaient comme refrains de brèves sentences populaires, rimés ou en forme de proverbes. Les troubadours provençaux appelaient cette séquence poétique « refrain », et ceci est à l'origine du mot espagnol « refrain ».

3. Signalons que l'étude des proverbes est l'objet d'une discipline connue sous le nom de parémiologie depuis les années soixante.

4. Traduction :[Le proverbe] est un dicton [...] populaire, sentencieux [...] et bref [...] de vérité attestée (proverbe), généralement symbolique (parabole) et exposé dans une forme poétique [...], qui contient une règle de conduite (adage) ou tout autre enseignement.

5. En wolof, le concept d'E.C. peut être rendu par celui de « Kàadu Cosaan ». Le vocable « Kàadu » pouvant se traduire par les termes français de « Parole », "Énoncé », «Propos », « Mot », et celui de «Cosaan" par ceux de "Genèse », "Origine », " Histoire », "Culture », "Valeurs de Civilisation ».

6. Pour toutes ces informations sur la société wolof, nous avons privilégié les ouvrages suivants : Barry, Boubacar, Le royaume du Walo : Le Sénégal avant la conquête, Paris, Karthala, 1985, p. 65-69, Malherbe Michel et Sall Cheikh, Parlons wolof: Langue et culture, Paris, Éditions L'Harmattan, 1989, p. 99-101. 
7. Kocc Barma Fall (1586-1655) avait quatre touffes de cheveux sur la tête et avait donné à chacune d'entre elles un nom propre. Ces noms représentaient chacun un aphorisme : 1-» Jiggen, soppal te bul wóolu » (Aime la femme, mais n'aie jamais confiance en elle) ; 2- «Buur du mbokk » (Le roi n'est jamais un parent) ; 3- «Doomu jittle du doom »; (L'enfant adoptif n'est jamais un vrai fils) ; 4- « Mag mat naa bàyyi cim réew » (Il est bon d'avoir un vieillard dans un village).

8. Dictionnaire Le Petit Robert de la langue française, Paris, 2004, pour la définition de la notion de vérité.

\section{RÉSUMÉS}

Appartenant à différents univers linguistiques, l'espagnol par notre formation universitaire, le français, la langue officielle de notre pays d'origine (Sénégal) et le wolof, notre langue maternelle, nous avons voulu, dans le cadre de nos recherches e, travailler sur un sujet où ces trois langues se côtoient d'un point de vue linguistique et culturel. Pour cela, nous avons choisi comme objet d'étude le proverbe car il existe dans toutes les langues et dans toutes les cultures et aussi parce qu'il appartient à la sagesse populaire. Le caractère multi-aspectuel du proverbe qui s'explique par la rencontre entre langue et culture (société) fait que son étude se situe au croisement de plusieurs domaines comme l'anthropologie, la sociologie, l'histoire... Á partir de ces trois langues (l'espagnol et le français, deux langues indoeuropéennes et le wolof, une langue nigéro-congolaise) et cultures, nous proposons dans cet article une approche théorique des proverbes espagnols, français et wolof à travers le concept d'énoncé culturel (E.C..) qui englobe l'ensemble des énoncés sentencieux de différentes langues, comme ici, les langues française, espagnole et wolof. Nous justifierons, dans cet article, le choix de cette notion et le sens que nous en retenons.

We belong to different world's language: Spanish, French and Wolof. We wanted to work on a subject where the three languages side by side a linguistic point of view and cultural. This work will be performed a comparative and contrastive. This is why we have chosen as an object of study as the proverb exists in all languages and in all cultures.

A from these three languages (Spanish and French, two Indo-European languages and Wolof, a language Niger-Congo), and cultures, we propose in this article we focus on a theoretical approach that allows us to work proverbs Spanish, French and Wolof adopting the concept cultural statement.

The adoption of this concept allows us to embrace all the different languages sententious statements data as is the case in this article with the French, Spanish and Wolof.

We will also see why we prefer the notion of cultural statement than saying, specifying what we mean by the notion of cultural statement.

\section{INDEX}

Mots-clés : autorité énonciative, parémie, proverbe, énoncé culturel, énoncé sentencieux

Keywords : cultural statements, proverbs, paremiology, French, spanish, wolof 
AUTEUR

MAME COUNA MBAYE

Université d'Artois 